\title{
Epidemic Dynamics of Malicious Code Detection Architecture in Critical Environment
}

\author{
A. Edwin Robert and M. Hemalatha* \\ Department of Computer Science, Karpagam University, Coimbatore, India; \\ edrt.edwin@gmail.com, csresearchhema@gmail.com
}

\begin{abstract}
In present world applications of software in other domains have their own privileges and their own control over other application also fulfilling their own testing methods acting as a tool in solving the given problem. Application integrity is highlighted in existing work but in our proposed approach MCBA (Malicious Code Behavior Analysis). In our proposed study the method of MCBA approach error correcting codes in the kernel is incorporated. Our objective is to incorporate a protection mechanism that saves the application even though the system's memory gets corrupted. In exploring the trusted MCBA Server to identify and monitor the portion of the system where corruption occurs and the server segregates the reason for various malicious impacts. Therefore, two approaches have been simulated: one is authenticated check and the next is unauthenticated check. In an authenticated check, a matching schema (e.g., the MCBA) applies dataset pattern recognition techniques to check malicious pattern by comparing it to the incoming applications during execution, if malicious packet is found it protects the system, in an unauthenticated check, the malicious packet from the guest OS for example root kits enters into our local system and it securely stores a cloned image of the guest OS memory at boot time, this method incorporates a VMM (Virtual Memory Monitor) and it allows only the instructions to read from the cloned copy of memory but never execute the instruction, though it is so, sometimes the instructions are malicious and it is unsecured. This paper emphasizes the MCBA architecture, incorporates monitoring, detecting and healing process which are so feasible to apply in real time environment ,it is very economically used for the technical programmers who are designing source code for various domains in Software market.
\end{abstract}

Keywords: Dataset, Error Correcting, Malicious, Matching, Privileges

\section{Introduction}

In presently used commercial operating systems, the trusted software applications executes and they are very complex preventing most of the security and testing methods in finding malicious packets.Vulnerable applications running in the same machine increases False positive rate of the system .In critical application in order to modify the kernel of the memory we need to take over the OS privilege. We aim to detect memory corruption especially in the local host. Moreover, if Local host is connected to the Remote host then the effect is very high. MaliciousOriented Recovery $(\mathrm{MOR})^{1}$ makes the applications that are affected by malicious code can be backed up by means of using the detection algorithms and filtering the benign files for automatic backup. The approach that we adopt in this paper is to use a trusted MCBA (Malicious Code Behavior Analysis Architecture) to detect and repair the corrupted application memory pages. As such, our work can be seen as a technique for transparently incorporating MOR into the execution environment without modifying the original application code. Existing research has already acknowledged the importance of protecting critical applications against an untrusted and/or compromised execution environment. The proposed approach is to employ a trusted MCBA that interacts between the OS and the malicious application, and restricts the kernel's access with respect to the memory space of the protected

${ }^{*}$ Author for correspondence 
application. These MCBA - based solutions generally fall into two broad categories: Authenticated check and Unauthenticated check.

\subsection{Authenticated Check}

In this category, a trusted component (e.g., the MCBA) applies cryptographic techniques to validate the integrity of the application's memory image during execution ${ }^{2}$. Virtual machines are isolated and then keeping the authentication check by using cryptographic hashing algorithm in prior to the incoming of untrusted applications from Remote systems. The specifications of the soft wares and hardwares running on the virtual machine can be known by implementing a private key ${ }^{3}$. Securing the critical applications running on the virtual server can be done by encrypting the memory specification. When the benign application runs, authentication is initiated and decryption process occurs.

\subsection{Unauthenticated Check ${ }^{4}$}

Incorporating the cloned image of the kernel we can save the OS from the external threats. As the system runs, only instructions retrieved from this copy are permitted to execute in kernel mode. That is, if a root kit has been installed after the system boots, it cannot execute, as its instructions do not exist in the protected space. Protecting the OS at runtime, it is not possible to protect safety critical applications if the original memory specification is malicious. Observe that these approaches primarily focus on detection of memory corruption. Authentication check terminates the protected application if corruption is detected. Memory duplication techniques detect and prevent the incoming of malicious code getting into memory drives, but do not protect application code. In vulnerable applications, detection of corruption alone is inadequate, as disruption of the execution may have disastrous consequences. There is no chance of detecting the vulnerable code again the intruder gets succeeded. In recovering benign process, authentication technique must be integrated with mechanisms for checking the current application ${ }^{5}$. Recovery and checking processes can be performed at the top layer providing confident to the OS in maintaining application integrity and granularity of recovery. Hence correct application is resuming from the previous authentication check.

\section{Related Systems}

Authentication of executed code has received a lot of attention in recent years, due to novel applications such as outsourcing of services ${ }^{6}$. By using a trusted platform module in the remote host instructions in the memory specifications are sequentially executed. In a trusted hardware application cryptographic hashes are used for storage confidentiality ${ }^{7}$. In real time applications preventing unauthorized applications and maintaining trusted OS applications can be incorporated by using database of hashes for the code and static data segments of user aware applications. In executing an incoming process, the OS checks if the executable's hash matches one of the values stored in the database, otherwise execution is prohibited. This type of application systems does not address memory corruption that occurs at runtime. Several solutions for secure code execution based on VMMs have been proposed $^{8}$. Trusted Virtual server of MCBA guarantees the integrity of system calls used by applications ${ }^{9}$. For protection and integrity of hardware pages in a commodity OS virtualization is done. Our proposed work focuses on creating a trusted environment in which the benign application is filtered from vulnerability and proceeds further.

\section{Proposed MCBA Architecture}

The goal of our design is to protect the code and data for applications executing within an untrusted OS environment. That is, we aim to provide a robust defensive mechanism that protects the application even if the OS kernel has been corrupted. Our goal is more ambitious, as we want to provide a probabilistic guarantee that the application can recover from memory corruption and continue processing.

Figure 1 shows a high-level view of proposed system. The guest OS executes within an untrusted zone (indicated by the hatched area) that consists of an emulated memory that is established by the trusted VMM. The guest OS has complete control over the emulated memory. Our goal is to execute trusted guest applications within this untrusted zone by leveraging the VMM. Before the guest OS boots, the VMM establishes a segment of protected physical memory that is beyond the reach of the guest OS. When a guest application runs, the VMM allocates a portion of this memory to store verification and reconstruction data (e.g., hashes, redundant blocks) that 


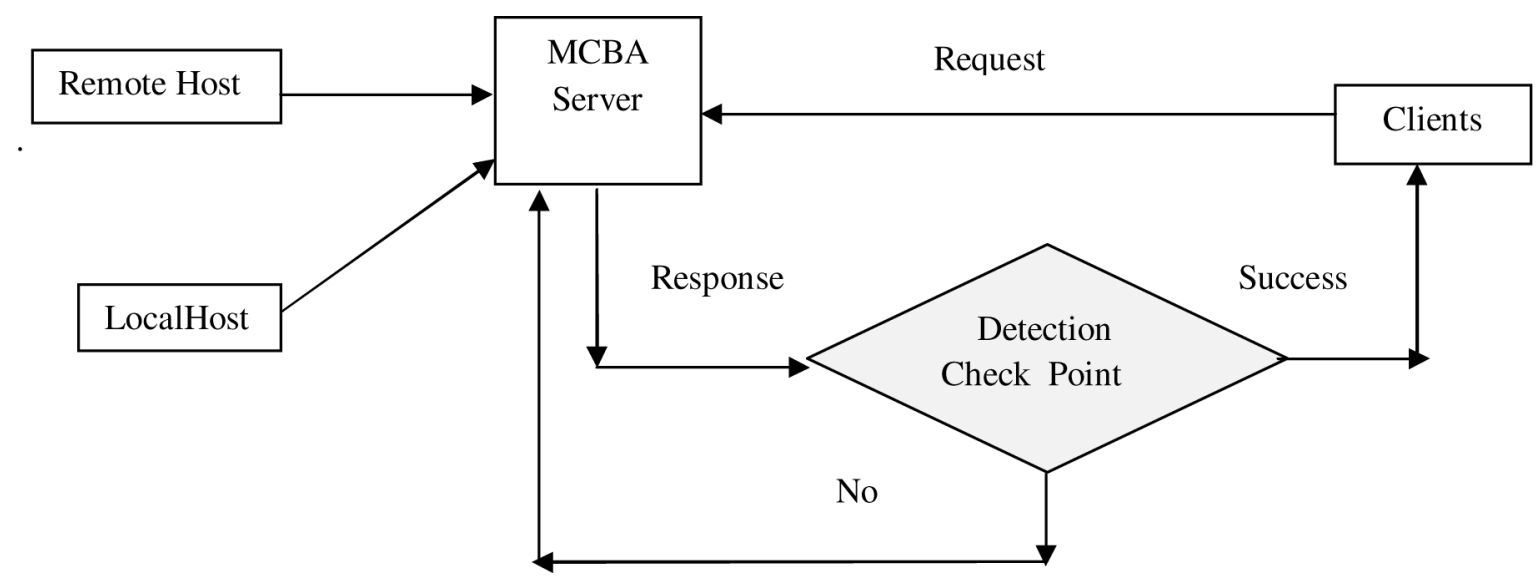

Figure 1. MCBA Architecture.

Table 1. Results of malware samples detected and estimated

\begin{tabular}{lcc}
\hline $\begin{array}{l}\text { Malware Types } \\
\text { Detected }\end{array}$ & $\begin{array}{c}\text { Packets From } \\
\text { Different OS }\end{array}$ & $\begin{array}{c}\text { Total Malwares } \\
\text { Found }\end{array}$ \\
\hline Boot Sector & & 50 \\
Blocking USB & & 30 \\
OS Shutdown & Windows 7/ & 92 \\
OS Crash & Windows XP/ & 100 \\
Remote Host & Windows & 60 \\
Exec File & 2000/ & 65 \\
Modify reg & Windows 98 & 80 \\
Copy Application & & 50 \\
Obtain System Info & & 45 \\
\hline
\end{tabular}

is specific to that application. Table 1 shows the malware samples detected and estimated.

Considering a trusted application running on a guest platform that also has a vulnerable network-facing application, such as an email client, our model is that an attacker exploits vulnerability in the untrusted application to inject code in the OS. The injected code then corrupts that application's page tables to point to the trusted application, and then modifies the trusted application's memory contents. Thus, the memory corruption originates from a source outside of the trusted application.

\subsection{Experimental Analysis of Virus Vulnerability Algorithm}

This application is tested by calculating the Fitness value using the Binominal expression to detect whether the code is immune from common vulnerabilities, such as buffer overflows. As such, we do not consider any attacks exploiting the application itself.

Table 1 shows how the malware types affect the different operating system by knowing the Malware and benign types in contradiction with normal test methods

$$
(x+a)^{n}=\sum_{k=0}^{n}\left(\begin{array}{l}
n \\
k
\end{array}\right) x^{k} a^{1-k}
$$

where, $\mathrm{x}$ is Malware samples and $\mathrm{n}$ is No. of detected Fitness values. Table 2 shows malware types segregated in different OS by Fitness values (Table 2).

As a description of a sample attack, consider a trusted application running on a guest platform that also has a vulnerable network-facing application, such as an email client. Our model detects the vulnerability in the untrusted application to inject code in the OS by knowing the fitness value. The injected code then corrupts that application's page tables to point to the trusted application, and then modifies the trusted application's memory contents. Thus, the memory corruption originates from a source outside of the trusted application. We also

Table 2. Malware types Segregated in different OS by Fitness values

\begin{tabular}{lccc}
\hline Operating System & $\begin{array}{c}\text { Malware } \\
\text { Type }\end{array}$ & $\begin{array}{c}\text { Fitness } \\
\text { Value }\end{array}$ & $\begin{array}{c}\text { Benign } \\
\text { Type }\end{array}$ \\
\hline Windows XP & Worm & 2.98 & Extreme \\
Windows NT & Trojan & 2.25 & Medium \\
Windows 98 & P2P worm & 1.32 & Very Low \\
Windows 2000 & Root Kit & 2.30 & Medium \\
Windows Server2003 & Back Door & 2.15 & Medium \\
Windows 7 & Boot Sector & 1.44 & Very Small \\
\hline
\end{tabular}


assume that the attacker corrupts only a small portion of the trusted application. This is consistent with scenarios where attackers aim to remain stealthy, while causing the application to deviate from the correct execution flow.

\subsection{On Shared Memory}

In an On Shared Memory indirect corruption is still possible. That is, the kernel could collude with a malicious user-mode application. It is achieved by storing the owner of the page, and enforcing a rule of no bidirectional shared memory writes. For example consider two applications Node $\mathrm{X}$ and Node $\mathrm{Y}$ that need to exchange data. If Node $\mathrm{X}$ wishes to send data to Node $\mathrm{Y}$, it would store the data in a page owned by Node X. Node Y could then read that data, but could not modify it. If Node $Y$ needed to respond in some way, it would write that data to a page that Node Y owns and Node X could later read. Thus, legitimate usermode applications that need to share data would have to cooperate accordingly.

\subsection{Vulnerability Analysis}

The stated goal is to protect trusted guest applications from memory corruption attacks launched by the guest OS or other guest applications.

This protection through the combination of the encoding scheme and verifying the integrity of encoded blocks with a cryptographic hash function.

While this design offers a robust layer of protection, a number of remaining threats have been identified. First, this system must trust the software that exists outside the trust boundary (Figure 2). Specifically, the VMM must be considered trusted. Also, if a host OS exists below the VMM, it must also be trusted ${ }^{15}$. In order for an attacker to corrupt a guest application successfully, he would first have to construct a collision under the hash algorithm. As these data structures are inaccessible to the guest OS and applications, the attacker would have to resort to guessing. Thus, we find successful corruption attacks from other guest applications to be extremely implausible, though possible in theory.

\subsection{Application Vulnerabilities}

To be thorough, the VMM, which is aware of the page directories, can track this and react accordingly. However, many environments require trusted applications that have undergone formal analysis and verification. Our design

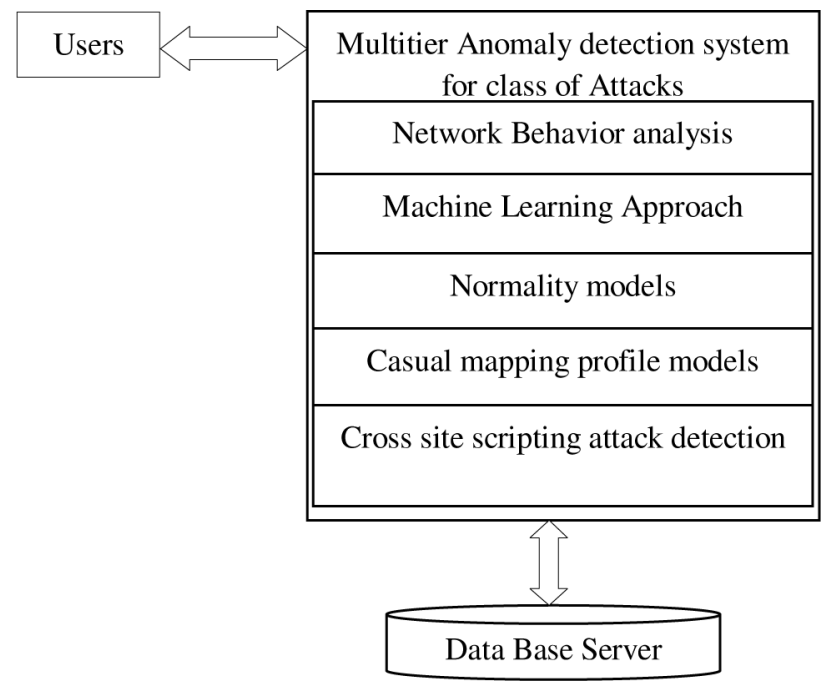

Figure 2. Architecture diagram of Denial of service.

offers these applications a resilient execution environment that protects the application from external threats.

$$
\text { Vulnerability }=\frac{\alpha * \beta \sqrt[4]{d * 4}}{\alpha} \text { Strength }
$$

where $\alpha$ is the external virus from the network and $\beta$ is corrupted virus due to internal virus affected the kernel. Also d is Corrupted network data. If the critical application sends or receives data across a network interface, the guest OS kernel has an opportunity to modify this data in transit ${ }^{12}$. Specifically, access to the network card is set up by the OS via a system call. In the case of verifying received data, a public key could be hard-coded into the application without incurring a significant threat. Securing private or symmetric keys, though, is not possible in our current approach, as the guest OS has full access to read that application's memory space and would be able to forge messages easily.

A scheme that provides a probabilistic guarantee of the latter is also proposed. This system, as described above, may not be able to recover transparently, especially if the amount of corruption within a single page is large.

Observe that, if the recovery fails, the MCBA is immediately aware of the failure, and knows exactly which page in the application's linear address space is corrupted. As the trusted component, the MCBA would have full access to replace the memory image as necessary. If the corrupted page consists of code or read-only data, the MCBA can extract the relevant portion of the application from a protected local storage that is inaccessible to the guest OS. 
Clearly, the MCBA has insufficient information to initiate a transparent local recovery (Figure 3 ).

\section{Implementation and Evaluation}

Evaluation can be incorporated by using a prototype has been implemented to measure the performance impact of our system design knowing the detection rate ${ }^{10,11}$. Whenever an operation causes a switch to authenticated mode the most recently used pages are encoded. In an On demand Scanning method decoding is done, whenever a page is first referenced during detecting and monitoring process.

While the system in Figure 2 assumes that the guest OS may be compromised, it is assumed that it is still somewhat functional. That is, if the attacker's goal is merely to shut down the system, he could corrupt the OS beyond repair ${ }^{13}$. This attack is outside the scope of our study, as focus is on scenarios where the attacker's goal is to keep the system working in a compromised fashion ${ }^{14}$. A more subtle attack could aim to prevent the OS from scheduling the trusted application. To defend against this attack, we suggest deploying a technique similar to a watchdog timer.
It provides more insight into the fitness value and detection rate implementations. The matches occurred in the Datasets can be given by $\mathrm{M}(\mathrm{N}-\mathrm{M}+1)$, because the pattern matched. Our test case generated a rate that one percent of pages are corrupted; consequently, while the MCBA encoding and decoding require significant time, this rarely occurs, yielding the low average time per packet. Table 3 provides Malware Detection Rate based on different OS specifications.

\section{Conclusion}

A novel approach MCBA is suggested for protecting applications against memory corruption attacks by

Table 3. Malware Detection Rate based on different OS specifications

\begin{tabular}{lcc}
\hline Operating System & $\begin{array}{c}\text { Types of Malware } \\
\text { Detected }\end{array}$ & $\begin{array}{c}\text { Detection } \\
\text { rate(\%) }\end{array}$ \\
\hline WindowsXP & OS Shutdown & 82.3 \\
WindowsNT & OS Crash & 23.1 \\
Windows 98 & Remote Host & 135.2 \\
Windows 2000 & Exec File & 189.2 \\
Windows Server2003 & Modify reg & 200.2 \\
\hline
\end{tabular}

$$
\text { Detection rate }=\frac{\text { True Positive }+ \text { True Negative }}{\text { True Positive }+ \text { True Negative }+ \text { False Positive }+ \text { False Negative }} * 100
$$

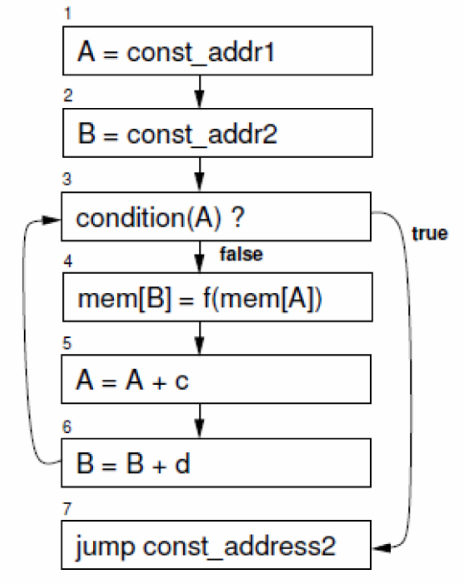

(a) Template of malicious behavior.

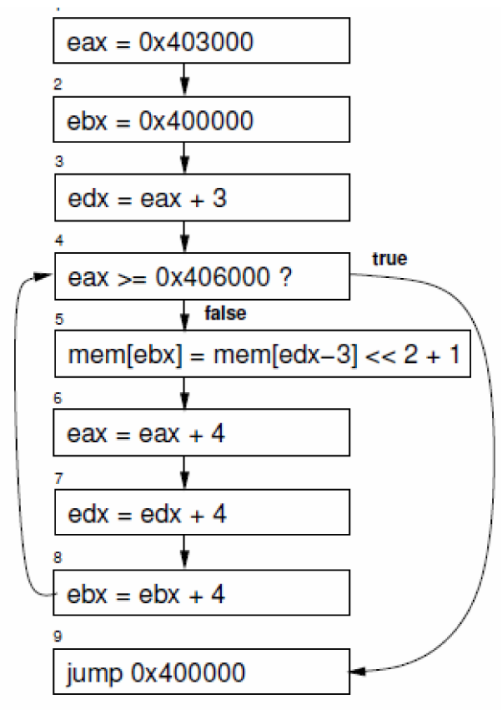

(b) Malware instance.

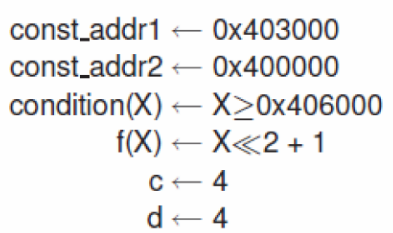

(c) Execution context.

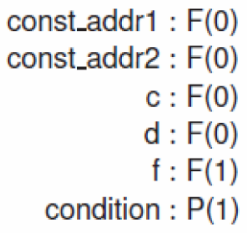

(d) Symbolic constant types.

Figure 3. MCBA Points towards the corrupted page in Memory Image application. 
incorporating efficient encoding of memory contents by static method. In this approach, it may be desirable to protect only critical portions, therefore reducing execution and protected storage overhead. Another direction would be to define the protocols and program analysis techniques for remote application recovery and other types of encoding schemes could be explored. In future enhancement, it is planned to provide MCBA-based protection mechanisms by means of neural networks in which the newly virus files are updated.

\section{References}

1. Garfinkel T, Pfaff B, Chow J, Rosenblum M, Boneh D. Terra: a virtual machine-based platform for trusted computing. Proc. 19th ACM Symp. Operating System Principles (SOSP); 2003. p. 193-206.

2. Chen X, Garfinkel T, Lewis EC, Subrahmanyam $P$, Waldspurger CA, Boneh D, Dwoskin J, Ports DRK. Overshadow: a virtualization-based approach to retrofitting protection in commodity operating systems. Proc. 13th Int'l Conf. Architectural Support for Programming Languages and Operating Systems (ASPLOS); 2008.

3. Riley R, Jiang X, Xu D. Guest-transparent prevention of kernel rootkits with vmm-based memory shadowing. Proc. 11th Int'l Symp Recent Advances in Intrusion Detection (RAID); 2008. p. 1-20.

4. Elnozahy ENM, Alvisi L, Wang Y-M, Johnson DB. A survey of rollback-recovery protocols in message-passing systems. ACM Computing Surveys. 2002; 34:375-408.

5. Reed IS, Solomon G. Polynomial codes over certain finite fields. J Soc for Industrial and Applied Math (SIAM). 1960; $8(2): 300-4$.
6. Wang Z, Jiang X. HyperSafe: a lightweight approach to provide lifetime hypervisor control-flow integrity. Proc IEEE Symp Security and Privacy; 2010.

7. Lie D, Thekkath CA, Horowitz M. Implementing an untrusted operating system on trusted hardware. Proc 19th ACM Symp Operating System Principles (SOSP). 2003; 178-92.

8. Piromsopa K, Enbody RJ. Secure bit: transparent, hardware buffer-overflow protection. IEEE Trans Dependable and Secure Computing. 2006; 3(4):365-76.

9. Sidiroglou S, Laadan O, Perez C, Viennot N, Nieh J, Keromytis AD. ASSURE: Automatic software self-healing using rescue points. Proc 14th Int'l Conf Architectural Support for Programming Languages and Operating Systems (ASPLOS). 2009; 37-48.

10. Litty L, Lagar-Cavilla HA, Lie D. Hypervisor support for identifying covertly executing binaries. Proc 17th USENIX Conf Security Symp. 2008; p. 243-58.

11. Wang Z, Jiang X, Cui W, Ning P. Countering kernel rootkits with lightweight hook protection. Proc 16th ACM Conf Computer and Comm Security (CCS). 2009; p. 545-54.

12. Xu J, Nakka N. Defeating memory corruption attacks via pointer taintedness detection. Proc International Systems and Networks. 2005; 378-87.

13. Fox A, Patterson D. Self-repairing computers. Scientific Am. 2003; 288:54-61.

14. Luby M. LT codes. Proc IEEE 43rd Ann Symp Foundations of Computer Science (FOCS). 2002; 271-80.

15. McCune JM, Parno BJ, Perrig A, Reiter MK, Isozaki $\mathrm{H}$. Flicker: An execution infrastructure for TCB minimization. ACM SIGOPS Operating System Rev. 2008; 42(4); 315-28. 